\title{
Restricting a compact action to an injective subfactor
}

\author{
ROBERTO LONGO† \\ Dipartimento di Matematica, Seconda Università di Roma 'Tor Vergata' \\ Via Orazio Raimondo, 00173 Rome, Italy
}

(Received 30 November 1987)

\section{Introduction}

Suppose we are given an action $\alpha: G \rightarrow$ Aut $(M)$ of a group $G$ on a factor $M$; a possible way to analyse $\alpha$ may be to look at the invariant components where the action becomes more tractable. This point of view naturally leads to the study of the injective invariant subalgebras (recall for instance the good properties shared by amenable discrete or compact actions in the hyperfinite case [14]).

Being optimistic, one may hope to pursue a program of the following kind:

(a) find a $\alpha$-invariant injective subfactor $R$,

(b) describe the restriction of $\alpha$ to $R$,

(c) relate the restriction $\alpha \mid R$ to the original action.

However, in this generality one find obstructions at the very beginning. As shown by Popa [17], if $M$ is the factor associated with the free group on countably many generators and $\alpha: \mathbb{Z} \rightarrow$ Aut $(M)$ is the action determined by the shift on the generators, there exists no non-trivial injective $\alpha$-invariant von Neumann sub-algebra of $M$.

Although positive results are known, up to cocycle perturbations, for aperiodic unimodular automorphisms of factors of type II, nothing is known even in the case of a periodic automorphism [15].

The purpose of this paper is to provide an equivariant form of our previous construction [12] that will basically achieve the above program in the case of a dominant action of a compact group $G$ on an infinite factor $M$.

Let us recall that a subfactor $R \subset M$ is said to be simple if

$$
R \vee J R J=B(\mathscr{H}),
$$

where $J$ is the modular conjugation of $M$ acting standardly on $\mathscr{H} \equiv L^{2}(M)$.

Given a dominant action $\alpha: G \rightarrow$ Aut $(M)$ of a compact group $G$ on an infinite factor $M$ with separable predual, we shall construct a simple injective $\alpha$-invariant subfactor $R$, (a).

Note that every action on $M$ is cocycle conjugate to a dominant one $[7,13]$, therefore point (a) in the infinite case always has a solution up to perturbation by a distinguished element in $H_{\alpha}^{1}(G ; M)$. Moreover the dominance condition is automatically satisfied in a number of cases, for example if $M^{\alpha^{\prime}} \cap M=\mathbb{C} 1$ and $M^{\alpha}$ is infinite.

† Supported in part by Ministero della Pubblica Istruzione and CNR-GNAFA. 
Moreover we shall construct an $\alpha$-invariant copy of the Cuntz algebra $0_{\infty}$ dense in $R$ where $\alpha$ restricts to an action of canonical type, namely $\alpha$ leaves invariant a generating Hilbert space in $0_{\infty}[8],(b)$.

It seems to be an interesting unsolved problem to decide whether $R=M$ if $M$ is injective or, more generally, whether $R$ is maximal injective for all $M$.

Simple subfactors are particularly suitable in our framework because of their crucial property in the determination of the isomorphisms of $M$ : at least in principle, the restriction $\alpha \mid R$ contains all the information in the original action, (c).

Furthermore, when $G$ is discrete abelian (thus $\hat{G}$ is compact) and $\alpha$ is dominant, a duality argument shows the existence of an $\alpha$-invariant injective von Neumann subalgebra that is $\alpha$-simple in a sense to be described, in particular $\alpha$ acts ergodically on the relative commutant $R^{\prime} \cap M$.

In the last section of this paper we shall make a computation on the cohomology group $H^{1}(M, N)$ of an inclusion of von Neumann algebras $M \subset N$ with separable predual. In the special case when $N$ is a crossed product of $M$ by a discrete action, we shall obtain that $H^{1}(M, N)=\{0\}$, i.e. all derivations $\delta: M \rightarrow N$ are inner, extending a result in [5] in the finite case. A general twisted derivation theorem [6] allows us to use only the existence of simple injective subfactors and not the full invariance Theorem 1.1 (that motivated the result).

The general question whether $H^{1}(M, N)=\{0\}$ in general remains open, but we hope our methods will be useful or give an insight for future research.

As a corollary, we complete the missing point in the description of unbounded derivations defined on the finite spectrum subalgebra given by a compact abelian group action in the case, for example, of a fixed point subalgebra with trivial relative commutant [3]. The corresponding result for non-abelian compact groups is technically complicated and probably needs an extension of our argument.

The section ends with an extension theorem for compact centrally ergodic states, an invariant version of the Hahn-Banach theorem for factor states.

\section{Invariant simple injective subfactors}

Let $\alpha: G \rightarrow$ Aut $(M)$ be an action of a compact group $G$ on a von Neumann algebra $M$. We shall say that $\alpha$ is dominant if the fixed point algebra $M^{\alpha}$ is properly infinite and the monoidal spectrum of $\alpha$ is complete (for every $\pi \in \hat{G}$ there exists an $\alpha$-invariant Hilbert space $H$ in $M$ such that $\alpha \mid H$ is equivalent to $\pi$ ) $[7,16]$.

Notice that the dominance condition is automatically satisfied in a number of cases, for example if $M^{\alpha}$ is properly infinite and there exists a commuting ergodic action $[1,13]$.

Denote by $0_{\infty}$ the Cuntz algebra generated by a separable infinite dimensional Hilbert space of isometries $H$ [8]; any unitary operator on $H$ gives rise to a canonical automorphism of $0_{\infty}$; if $G$ is a topological group, any strongly continuous unitary representation of $G$ on $H$ determines a pointwise norm continuous action of $G$ on $0_{\infty}$ that we shall refer to as an action of canonical type.

The next theorem has a natural extension to the case of a non-trivial centre; for simplicity we restrict ourselves to the factor case. 
THEOREM 2.1. Let $M$ be an infinite factor with separable predual and $\alpha: G \rightarrow$ Aut $(M)$ a dominant action of a compact group $G$ on $M$.

There exists a simple injective $\alpha$-invariant subfactor $R$ of $M$.

Moreover $R$ contains a dense $\alpha$-invariant copy of $0_{\infty}$ and the restriction of $\alpha$ to $0_{\infty}$ is an action of canonical type.

The proof of this theorem is postponed to the next section.

Corollary 2.2. Let $\alpha: G \rightarrow$ Aut $(M)$ be an action of a compact group on an infinite factor $M$. There exists an action $\beta: G \rightarrow$ Aut $(M)$ cocycle conjugate to $\alpha$, that normalizes a simple injective subfactor of $M$, provided $G$ and $M_{*}$ are separable.

Proof. Since any action $\alpha: G \rightarrow$ Aut $(M)$ as above may be perturbed to a dominant one [13] we are in the situation covered by Theorem 2.1 .

In view of the following corollary we introduce some notations. Let $R \subset M$ be an inclusion of von Neumann algebras, where $M$ acts standardly on $L^{2}(M)$ with modular conjugation $J$. Setting $S \equiv J R^{\prime} J$, the three term inclusion

$$
R \subset M \subset S
$$

does not depend on $J$, up to isomorphism, and $R$ is simple in $M$ iff $R^{\prime} \cap S=\mathbb{C} 1$. Given $\theta \in$ Aut $(M, R)$ (that is $\theta \in$ Aut $(M)$ and $\theta(R)=R$ ) the unitary standard implementation of $\theta$ implements a canonical extension of $\theta$ in Aut $(S)$, still denoted by $\theta$. Given a group action $\theta: \Gamma \rightarrow$ Aut $(M, R)$, we shall say that $R$ is $\theta$-simple if $\theta$ acts ergodically on $R^{\prime} \cap S$ and for any given $p \in \Gamma, p \neq e$,

$$
v \in S^{\theta}, \quad \theta_{p}(x) v=v x \text { for all } x \in R \Rightarrow v=0
$$

$(\theta \mid R$ is free with respect to $S)$.

COROLlary 2.3. Let $N$ be an infinite factor with separable predual and $\theta \mid \Gamma \rightarrow$ Aut $(N)$ a dominant outer action of a discrete abelian group $\Gamma$. There exists a $\theta$-invariant $\theta$-simple injective von Neumann subalgebra $R_{0}$ of $N$.

Proof. Let $M \equiv N \times_{\theta} \Gamma$ be the crossed product of $N$ by $\theta$ and $\alpha: G \rightarrow$ Aut $(M)$ the dual action of $G \equiv \hat{\Gamma}$. Let $R \subset M$ be the simple injective $\alpha$-invariant subfactor given by Theorem 1.1 and denote $R_{0} \equiv N \cap R$ its $\alpha$-fixed point algebra. The restriction of $\alpha$ to $R$ is dominant by construction (cf. [9, Corollary 9.7]) therefore the inclusion $R \subset M$ is the crossed product of $R_{0} \subset N$ by an action $\theta^{\prime}: \Gamma \rightarrow$ Aut $\left(N, R_{0}\right)$ conjugate to $\theta$ by a cocycle in $N$. By perturbing $\theta^{\prime}$ by a cocycle in $N$, we may assume that $\theta^{\prime} \mid R$ is dominant. Since $\theta$ and $\theta^{\prime}$ are both dominant, they are conjugate [7] and we may replace $\theta^{\prime}$ by $\theta$ in our considerations. With $S$ the canonical extension of $R \subset N$ as before and $S_{0} \equiv S \cap M$, the corollary follows by the following lemma because $R^{\prime} \cap S=\mathbb{C} 1$.

LEMMA 2.4. Let $R_{0} \subset S_{0}$ be an inclusion of von Neumann algebras, $\theta: \Gamma \rightarrow$ Aut $\left(S_{0}, R_{0}\right)$ and action of a discrete group and $R \equiv R_{0} \times_{\theta} \Gamma \subset S \equiv S_{0} \times_{\theta} \Gamma$ the crossed product inclusion. The elements of $R^{\prime} \cap S$ are those $x \in S$ with Fourier expansion

$$
x=\sum_{t \in \Gamma} c_{t} U_{t}, \quad c_{t} \in S
$$


such that

$$
\theta_{s}\left(c_{t}\right)=c_{s t s}{ }^{-1}, \quad c_{t} \theta_{t}(a)=a c_{t}, \quad a \in R_{0}, \quad t, s \in \Gamma .
$$

Proof. By the unicity of the Fourier expansion, the proof is obtained by simply imposing the commutation condition.

Note that Lemma 2.4 provides another motivation for studying local charges [9]. Remark. Although Theorem 2.1 applies to infinite factors only, one may notice that if $M$ is any factor with separable predual and $\alpha: G \rightarrow$ Aut $(M)$ is an action of a compact group $G$, then $\alpha \otimes$ ad $(\lambda) \otimes$ id (where $\lambda$ denotes the regular representation of $G)$ is a dominant action of $G$ on $M \otimes B\left(L^{2}(G)\right) \otimes B\left(l^{2}(\mathbb{N})\right)$ that normalizes a simple injective subfactor.

\section{Invariant standard states}

Let $M$ be a von Neumann algebra, $\alpha: G \rightarrow$ Aut $(M)$ an action of a compact group and $\omega$ a faithful $\alpha$-invariant state of $M$. We assume $\omega$ is given by a cyclic separating vector $\Omega$ on the underlying Hilbert space $\mathscr{H}$ and denote by $U$ the $\Omega$-fixing unitary representation of $G$ on $\mathscr{H}$ implementing $\alpha$ and by $\varepsilon_{\pi}$ (respectively $E_{\pi}$ ) the projection of $M$ (respectively $\mathscr{H}$ ) onto the spectral subspace $M_{n}$ (respectively $\mathscr{H}_{\pi}$ ) associated with the irreducible representation $\pi \in G$, namely

$$
\begin{aligned}
\varepsilon_{\pi} & \equiv \int \overline{\chi_{\pi}(g)} \alpha_{g} d g, \\
E_{\pi} & \equiv \int \overline{\chi_{\pi}(g)} U_{\mathrm{g}} d g,
\end{aligned}
$$

where $\chi_{\pi}$ is the normalized character of $\pi$. It follows that

$$
E_{\pi} x \Omega=\varepsilon_{\pi}(x) \Omega
$$

thus

$$
E_{\pi}=\left[M_{\pi} \Omega\right] \text {. }
$$

Now suppose $H \subset M$ is an $\alpha$-invariant Hilbert space in $M$ and the restriction of $\alpha$ to $H$ is equivalent to $\pi$. With $\left\{v_{i}, i \in I\right\}$ an orthonormal basis of $H$ one has [16]

$$
E_{\bar{\pi}}=d \sum_{i} v_{i}^{*} E_{0} v_{i}
$$

where $d$ is the dimension of $\pi$ and $E_{0} \equiv\left[M^{\alpha} \Omega\right]$ is the projection onto the $U$-invariant vectors. In fact for $x \in M$ one has

$$
\begin{aligned}
E_{\pi} x \Omega & =\varepsilon_{\pi}(x) \Omega=\int \overline{\chi_{\pi}(g)} \alpha_{g}(x) d g \Omega \\
& =\int d \sum_{i} v_{i}^{*} \alpha_{g}\left(v_{i}\right) \alpha_{g}(x) d g \Omega \\
& =d \sum_{i} v_{i}^{*} \int \alpha_{g}\left(v_{i} x\right) d g \Omega \\
& =d \sum_{i} v_{i}^{*} E_{0} v_{i} x \Omega .
\end{aligned}
$$


Recall now that a standard state $\omega$ for an inclusion $N \subset M$ of von Neumann algebras is a faithful normal state of $M$ represented by a vector $\Omega \in L^{2}(M)$ cyclic and separating for $N, M$ and $N^{\prime} \cap M$ ( $\Omega$ is a standard vector).

The following proposition is contained in [4].

Proposition 3.1. Let $\alpha: G \rightarrow$ Aut $(M)$ be an action of a compact group $G$ on a von Neumann algebra $M$ and $N$ an $\alpha$-invariant von Neumann subalgebra. If $\alpha \mid N$ and $\alpha \mid N^{\prime} \cap M$ are both dominant, then every standard state $\omega_{0}$ for both $N^{\alpha} \subset M^{\alpha}$ and $\left(N^{\prime} \cap M\right)^{\alpha} \subset M^{\alpha}$ extends to an $\alpha$-invariant standard state $\omega$ for $N \subset M$.

Proof. Let $\varepsilon: M \rightarrow M^{\alpha}$ be the usual conditional expectation and put $\omega \equiv \omega_{0} \circ \varepsilon$; then $\omega$ is faithful and $\alpha$-invariant. Let $\Omega \in \mathscr{H}\left(=L^{2}(M)\right)$ be a cyclic vector representative of $\omega$; since $\omega$ is $\alpha$-invariant we may choose $\Omega \in L^{2}\left(M^{\alpha}\right)\left(=E_{0} \mathscr{H}\right)$. By assumptions $\Omega$ is a standard vector for $N^{\alpha} \subset M^{\alpha}$. We have to show that $\Omega$ is cyclic for $N$ and $N^{\prime} \cap M$. Since $\mathscr{H}$ is spanned by the $\mathscr{H}_{\pi}, \pi \in \hat{G}$, it is enough to show that

$$
[N \Omega] \geq E_{\pi}, \quad\left[\left(N^{\prime} \cap M\right) \Omega\right] \geq E_{\pi}, \quad \pi \in \hat{G} .
$$

Let $H \subset N$ be an $\alpha$-invariant Hilbert space of class $\pi$ with orthonormal basis $\left\{v_{i}, i \in I\right\}$; since $E_{0}=\left[M^{\alpha} \Omega\right]=\left[N^{\alpha} \Omega\right]$, we have

$$
\left[N_{\pi} \Omega\right]=d \sum_{i} v_{i}^{*} E_{0} v_{i}=E_{\pi}
$$

therefore $[N \Omega] \geq E_{\pi}$. Analogously if $H$ was chosen in $N^{\prime} \cap M$, we would have

$$
\left[\left(N^{\prime} \cap M\right)_{\pi} \Omega\right]=d \sum_{i} v_{i}^{*} E_{0} v_{i}=E_{\pi}
$$

by using the fact that $\left[\left(N^{\prime} \cap M\right)^{\alpha} \Omega\right]=E_{0}$. It follows that $\Omega$ is a standard vector.

Corollary 3.2. Let $\alpha: G \rightarrow$ Aut $(M, N)$ be an action of a compact group $G$ on the inclusion of von Neumann algebras $N \subset M$. Assume $M_{*}$ is separable. If $\alpha \mid N$ and $\alpha \mid N^{\prime} \cap M$ are dominant, there exists an $\alpha$-invariant standard state for $N \subset M$.

Proof. Since $\alpha$ is dominant $N^{\alpha}$ and $\left(N^{\prime} \cap M\right)^{\alpha}$ are properly infinite. By the DixmierMarechal theorem as in [9, Proposition 1.2] there exists a common standard state for $N^{\alpha} \subset M^{\alpha}$ and $\left(N^{\prime} \cap M\right)^{\alpha} \subset M^{\alpha}$ and we apply Proposition 3.1.

Lemma 3.3. Let $M$ be $a$ von Neumann algebra with separable predual and $\alpha: G \rightarrow$ Aut $(M)$ a dominant action of a compact group. There exists an $\alpha$-invariant type $I$ subfactor $N \subset M$ and an $\alpha$-invariant standard state $\omega$ for $N \subset M$.

Proof. By Corollary 3.2 it is enough to construct an invariant type $I$ subfactor $N$ such that $\alpha \mid N$ and $\alpha \mid N^{\prime} \cap M$ are dominant. We shall first construct a type $I$ factor $F \supset M$, an extension of $\alpha$ to $F$ and an $\alpha$-invariant standard state $\omega$ for $M \subset F$. Setting $N \equiv \gamma_{\omega}(F)$ where $\gamma_{\omega}: F \rightarrow M$ is the associated canonical endomorphism [12], then $\omega \mid M$ will be $\alpha$-invariant; since $\gamma_{\omega}$ commutes with $\alpha, N$ will also be $\alpha$-invariant and $\omega$ is standard because of the identity

$$
N^{\prime} \cap M=\gamma_{\omega}(F)^{\prime} \cap M=J_{M} F J_{M} \cap M=J_{M}\left(F^{\prime} \cap M\right) J_{M}
$$

(with obvious notations). 
To construct $F$ we let $M$ act standardly on $\mathscr{H}=L^{2}(M)$ and put $F \equiv B(\mathscr{H})$. Let $U$ be the standard implementation of $\alpha$ and denote by the same symbol $\alpha$ the action of $G$ on $F$ implemented by $U$. Since $U$ commutes with the modular conjugation $J$ of $M$, then $\alpha \mid M^{\prime}$ is antiisomorphic to $\alpha \mid M$ by the Tomita commutation theorem. Since $\alpha \mid M$ is dominant by assumption, also $\alpha \mid\left(M^{\prime} \cap F\right)=M^{\prime}$ is dominant.

COROLlARY 3.4. Every compact metrizable group $G$ arises as a closed subgroup of Aut $(\Lambda)$ for some standard split $W^{*}$-inclusion $\Lambda$.

Proof. Let $\alpha=\operatorname{ad}(\lambda)$ on $B\left(L^{2}(G)\right)$ where $\lambda$ is the regular representation of $G$. Then $\alpha \otimes$ id on $B\left(L^{2}(G)\right) \otimes F$ is dominant if $F$ is a type $I_{\infty}$ factor. By Lemma 3.3 we conclude.

Proof of Theorem 2.1. Choose by Lemma 2.3 a standard inclusion of von Neumann algebras $(N, M, \Omega)$ with $N$ a type I $\alpha$-invariant subfactor and $\omega$ an $\alpha$-invariant standard state. The canonical endomorphism $\gamma_{\omega}: M \rightarrow N^{\prime} \cap M$ is inner [12, Theorem 3.1] and commutes with $\alpha$ (see the Appendix). The Hilbert space $H$ in $M$ implementing $\gamma_{\omega}$ is therefore $\alpha$-invariant as well as the $C^{*}$-algebra $0_{\infty}$ generated by $H$ and its weak closure $R$ which is a simple injective subfactor of $M$ [12]. In other words the construction of $R$ is made by the following canonical steps

$$
\begin{gathered}
(N, M, \omega) \\
\downarrow \\
\gamma_{\omega}: M \rightarrow N^{\prime} \cap M \\
\downarrow \\
H \equiv\left\{x \in M, \gamma_{\omega}(x) v=v x\right\} \\
\downarrow \\
0_{\infty} \equiv C^{*}(H) \\
\downarrow \\
R \equiv 0_{\infty}^{\prime \prime}
\end{gathered}
$$

and the $\alpha$-invariance of the initial data $(N, M, \omega)$ automatically gives the $\alpha$ invariance of the rest.

Remark. It would be interesting to extend Theorem 2.1 to the case of a locally compact group $G$. By considering invariant 'standard weights', cf [4], the key problem stays in the verification of the ergodic properties of the canonical endomorphism associated with a standard weight, a problem of independent interest.

\section{A cohomological calculation and further consequences}

An interesting problem in von Neumann algebras is to decide whether $H^{1}(M, N)=\{0\}$ for an inclusion of von Neumann algebras $M \subset N$ i.e. every derivation $\delta: M \rightarrow N$ is inner. In this section we consider a particular class of inclusions, where $N$ is the cross product of $M$ by a discrete action, and show that indeed $H^{1}(M, N)=\{0\}$ in this case, extending to the infinite case a result of Christensen [5]. We make use of the existence of simple injective subfactors and the Christensen-Evans derivation theorem [6]. 
Let then $\Gamma$ be a discrete group acting on the von Neumann algebra $M, \theta: \Gamma \rightarrow$ Aut $(M)$, and assume $M_{*}$ is separable; denote by $U_{t}, t \in \Gamma$, the usual unitaries in $N \equiv M \times_{\theta} \Gamma$ and put

$$
M_{t} \equiv M U_{t}=\left\{x U_{t}, x \in M\right\}, \quad t \in \Gamma .
$$

Then $M_{t}$ is naturally a normal $M$-bimodule isomorphic to $M$ with usual left multiplication and with twisted right multiplication $x \cdot y \equiv x \theta_{t}(y)$. With $\varepsilon: N \rightarrow M$ the usual conditional expectation, denote by $\varepsilon_{t}: N \rightarrow M_{t}$ the projections

$$
\varepsilon_{t}(x) \equiv \varepsilon\left(x U_{t}^{*}\right) U_{t} \quad x \in N .
$$

If $\delta: M \rightarrow N$ is a derivation, the maps

$$
\delta_{t}: M \rightarrow M_{t}, \quad \delta_{t} \equiv \varepsilon_{t} \circ \delta,
$$

are derivations since

$$
\begin{aligned}
\delta_{t}(x y) & =\varepsilon_{t}(\delta(x y))=\varepsilon_{t}(\delta(x) y)+\varepsilon_{t}(x \delta(y)) \\
& =\varepsilon_{t}(\delta(x)) y+x \varepsilon_{t}(\delta(y))=\delta_{t}(x) y+x \delta_{t}(y) \quad x, y \in M,
\end{aligned}
$$

By [6, Corollary 2.3], $H^{1}\left(M, M_{t}\right)=\{0\}$ thus the $\delta_{t}$ are inner.

Proposition 4.1. Let $M$ be a von Neumann algebra with separable predual and $N \equiv M \times{ }_{\theta} \Gamma$ the crossed product of $M$ by an action of a discrete group $\Gamma$. Every derivation $\delta: M \rightarrow N$ is inner.

Proof. We follow the above notations and assume for simplicity that $M$ is a factor and $\theta$ is outer. We may also assume $M$ is infinite [5]. Let $R \subset M$ be a simple injective subfactor; since $R$ is amenable [10] there exists $k \in N$ that implements $\delta \mid R$

$$
\delta(x)=[x, k], \quad x \in R
$$

and let

$$
k=\sum_{t \in \Gamma} k_{t}, \quad k_{t} \in M_{t}
$$

its Fourier expansion, where $k_{t} \equiv \varepsilon_{t}(k)$. It follows that $\delta_{t} \mid R$ is implemented by $k_{t}$

$$
\delta_{t}(x)=\varepsilon_{t} \circ \delta(x)=\varepsilon_{t}(x k-k x)=x k_{t}-k_{t} x, \quad x \in R .
$$

By [6] there exists $h_{t} \in N$ that implements $\delta_{t}$

$$
\delta_{t}(x)=x h_{t}-h_{t} x, \quad x \in M,
$$

therefore

$$
\left[x, h_{t}-k_{t}\right]=\delta_{t}(x)-\delta_{t}(x)=0, \quad x \in R .
$$

Since $R^{\prime} \cap N=\mathbb{C} 1$ [12, Theorem 4.8], we have $h_{t}=k_{t}+\lambda_{t}$ with $\lambda_{t} \in \mathbb{C}$, hence $k_{t}$ implements $\delta_{t}$ on $M$. In other words

$$
\varepsilon_{t}(\delta(x))=\left[x, k_{t}\right]=\varepsilon_{t}([x, k]), \quad x \in M
$$

and this implies

$$
\delta(x)=[x, k], \quad x \in M
$$

Remark. Further calculations on $H^{\prime}(M, N)$ are possible: if there exist injective von Neumann algebras, $R, S$ with $R \subset M \subset N \subset S$ and $R^{\prime} \cap S \subset N$ then the proof of [12, Corollary 4.10] carries on to entail the vanishing of $H^{\prime}(M, N)$. For example 
this is the case of the inclusion $A \otimes 1 \subset A \otimes B$ with $B$ injective, $B_{*}$ separable and $A$ properly infinite.

COROllary 4.2. Let $G$ be a compact abelian group and $\alpha: G \rightarrow$ Aut $(M)$ a dominiant action on a von Neumann algebra $M$ with separable predual. Every derivation $\delta: M^{\alpha} \rightarrow$ $M$ is inner.

Proof. Immediate by duality.

The above corollary furnishes the missing step in the following theorem of Bratteli and Goodman [3]. In this respect it would be interesting to extend it to the case of non abelian compact groups.

Denote below by $M_{F}$ the subalgebra of elements with finite spectrum.

Corollary 4.3. Let $M$ be a factor with separable predual and $\alpha: G \rightarrow$ Aut $(M)$ an action of a compact abelian group with $M^{\alpha^{\prime}} \cap M=\mathbb{C} 1$. If $\delta: M_{F} \rightarrow M$ is a derivation, then $\delta$ is closable and its closure is a generator. Moreover $\delta$ has a unique decomposition

$$
\delta=\delta_{0}+\delta_{1}
$$

when $\delta_{0}$ has a closure generating a one-parameter subgroup of $\alpha_{G}$ and $\delta_{1}$ is inner.

Proof. By [3] it is enough to show that $H^{1}\left(M^{\alpha}, M\right)=\{0\}$, that is true by the above corollary (by tensoring with $B(\mathscr{H})$ we may assume $M^{\alpha}$ infinite).

We end this section by proving a compact equivariant form of the factor state extension theorem.

Let $\alpha: G \rightarrow$ Aut $(A)$ an action of a compact group on $C^{*}$-algebras $A$. Recall that a state $\phi$ of $A$ is said to be centrally ergodic if the natural action of $G$ on the centre of $\pi_{\phi}(A)^{\prime \prime}$ is ergodic, i.e. $\left(\cdot \xi_{\phi}, \xi_{\phi}\right)$ is an extremal invariant state of $\pi_{\phi}(A) \vee \pi_{\phi}(A)^{\prime}$.

Propositron 4.4. Let $\alpha: G \rightarrow$ Aut $(A)$ be an action of a metrizable compact group $G$ on the $C^{*}$-algebra $A$ and $B \subset A$ a separable $\alpha$-invariant $C^{*}$-subalgebra. Every centrally ergodic state $\phi$ of $B$ extends to a centrally ergodic state of $A$.

Proof. The proof is obtained by making equivariant the argument in [11], therefore we only indicate the main variations. By tensoring $A$ with a suitable $C^{*}$-algebra, we may assume that $\pi_{\phi}(B)^{\prime}$ is type III and the adjoint action of $G$ on $\pi_{\phi}(B)^{\prime}$ is dominant. Then one chooses a simple $\alpha$-invariant injective von Neumann subalgebra of $\pi_{\phi}(B)^{\prime}$ and uses the Krein-Milman theorem. For more details see [2].

\section{Appendix}

We outline here a shorter and more transparent proof of the implication

$$
A \subset B \text { split } \Rightarrow \gamma_{\omega}: B \rightarrow A^{\prime} \cap B \text { inner }
$$

in [12, Theorem 3.1] on which our results rely (we use the notations in [12]).

By the split property the inclusion $A \subset B$ is isomorphic to $A \otimes 1 \subset B(\mathscr{H}) \otimes B$ $\left(\mathscr{H}=L^{2}(B)\right)$, hence we may identify $A^{\prime} \cap B \subset B$ with $A^{\prime} \otimes B \subset B(\mathscr{H}) \otimes B$, see [18].

By the Radon-Nikodym property [12, Theorem 1.1] the innerness of $\gamma_{\omega}$ does not depend on $\omega$; in particular we may let $\omega$ have the form $\omega=\phi \otimes \psi, \phi \in B(\mathscr{H})_{*}^{+}$, $\psi \in B_{*}^{+}$, so that $\gamma_{\omega}=\gamma_{\phi} \otimes \gamma_{\psi}=\gamma_{\phi} \otimes$ id. 
Since every endomorphism of $B(\mathscr{H})$ is inner (see $[12, \S 2]) \gamma_{\omega}$ is inner, hence $\gamma_{\omega}=\gamma_{\phi} \otimes \mathrm{id}$ is inner.

Acknowledgments. The author thanks D. Evans for the warm hospitality extended to him at the Warwick symposium on Operator Algebras in June-July 1987 where part of this paper was written.

\section{REFERENCES}

[1] H. Araki, R. Haag, D. Kastler \& M. Takesaki. Extension of states and chemical potential. Commun. Math. Phys. 53 (1977), 97-134.

[2] C. J. K. Batty. G-central subalgebras and extension of KMS states. J. Functional Anal. 66 (1986), 11-20.

[3] O. Bratteli \& F. M. Goodman, Derivations tangential to compact group actions: spectral conditions in the weak closure. Can. J. Math. 37 (1985), 160-192.

[4] D. Buchholz, S. Doplicher \& R. Longo. In preparation.

[5] E. Christensen. Extension of derivations. J. Functional Anal. 27 (1978), 234-247.

[6] E. Christensen \& D. E. Evans. Cohomology of operator algebras and quantum dynamical semigroups. J. London Math. Soc. 220 (1979), 358-368.

[7] A. Connes \& M. Takesaki. The flow of weights on a factor of type III. Tohoku Math. J. 29 (1977), 473-575.

[8] J. Cuntz. Simple $C^{*}$-algebras generated by isometries. Commun. Math. Phys. 57 (1977), 173-185.

[9] S. Doplicher \& R. Longo. Standard and split inclusions of von Neumann algebras. Invent. Math. 75 (1984), 493-536.

[10] R. V. Kadison. Derivations of operator algebras. Ann. of Math. 83 (1966), 280-293.

[11] R. Longo. Solution of the factorial Stone-Weierstrass conjecture. Invent. Math. 76 (1984), 145-155.

[12] R. Longo. Simple injective subfactors. Adv. in Math. 63 (1987), 152-171.

[13] Y. Nakagami \& M. Takesaki. Duality for crossed products of von Neumann algebras. Lecture Notes in Mathematics N. 731, Springer-Verlag: Berlin-Heidelberg, 1979.

[14] A. Ocneanu. Actions of amenable groups on factors. Lecture Notes in Mathematics 1138, SpringerVerlag: Berlin-Heidelberg, 1980. V. F. R. Jones \& M. Takesaki, Actions of compact abelian groups on semifinite factors. Acta Math. 153 (1984), 213-258.

[15] S. Popa, Hyperfinite subalgebras normalized by a given automorphism and related problems, Lecture Notes in Mathematics, 1132, pp. 421-433. Springer-Verlag. Berlin-Heidelberg, 1985.

[16] J. E. Roberts. Cross products of von Neumann algebras by group duals, Simpos. Math. 20 (1976), 335-363.

[17] S. Popa. Maximal abelian injective subalgebras in factors associated with free groups. Adv. in Math. S0 (1983), 27-48.

[18] C. D'Antoni \& R. Longo. Interpolation by type I factors and the flip automorphism. J. Functional Anal. 51 (1983), 361-371. 